\title{
Short OReport Mesenteric haematoma or tear is the criminal
}

\author{
Alireza Hamidian Jahromi, Asser M. Youssef
}

Department of Surgery, Louisiana State University Health Sciences Center, Shreveport, LA, USA

\section{Correspondence:}

Dr. Asser M. Youssef,

Email:ayouss@lsuhsc.edu aaseryou@gmail.com
We read with interest the report by Lalwani et al. from New Delhi, India reporting 8 cases of delayed/late presentation of small bowel (SB) stricture following blunt abdominal trauma (BAT). ${ }^{1}$ Eight cases being reported from a single centre is an indication that this pathological phenomenon is more common than it has previously been described. Lalwani et al. have not mentioned the exact number of cases of BAT that were reviewed between 1996 and 2008 at their centre, which if known would help in estimating the incidence of delayed mesenteric stricture following BAT. We report here a case of a young man who had a delayed presentation of SB obstruction secondary to a stricture 16 days following a motorcycle crash. ${ }^{2}$ In our case, the initial computed tomography (CT) scan showed that the patient had a mesenteric haematoma, which was managed conservatively. The repeat enhanced-CT scan 16 days posttrauma - when the patient presented with vomiting and increased abdominal pain — showed a stenotic loop of distal ileum adjacent to an expanded mesenteric mass. Pathological evaluation following exploratory laparotomy and resection of the loop revealed a loop of stenotic SB with oedematous mesentery, mucosal atrophy, ulcerations and serosal fibrous adhesions which indicate ischaemic changes. We have found and referenced 12 other reports (mainly case reports) of delayed SB obstruction after BAT from the medical literature. ${ }^{2}$ Since then we have also reported a different case of a young man who had a delayed presentation ( 5 weeks) of SB perforation following a BAT (motorcycle accident). ${ }^{3}$ It is of note to mention that this patient was also found to have a mesenteric haematoma on initial abdominal CT scan which was managed conservatively. An exploratory laparotomy at the time of delayed presentation (peritonitis) 5 weeks after the initial trauma showed a perforated thickened loop of the ileum forming a phlegmon in the lower abdomen. In the English medical literature, we found only 8 other reports of delayed post-traumatic presentation of ileal/jejunal perforation following BAT. ${ }^{3}$ The fact that there are very few reported cases in the literature for both the complications (SB stricture and/or perforation following BAT), is an evidence that these pathologies are not well represented and reported in the literature. A shared feature in the majority of the reported cases in the literature is the presence of minor tears and contusions of the mesentery, and/or mesenteric haematoma. Direct mechanical intestinal injury is unlikely to be the cause in situations where delayed presentation of SB stricture or perforation lags a few weeks or even months post-trauma. It is also unrealistic that an initial post-traumatic SB perforation goes undiagnosed for such a long period of time. In the above-mentioned case series of 8 patients with delayed post-traumatic SB stricture reported by Lalwani et al., ${ }^{1}$ the authors have reported a history of mesenteric tear repair in one of the patients, diagnostic laparoscopy in the second patient, and laparotomy with lysis of adhesion in the third patient (highly likely to cause mesenteric tears). There was also a reported case (fourth patient) with a thrombus involving the portal vein, extending into the superior mesenteric vein which could affect the blood circulation in the SB mesentery. Although the immediate post-traumatic abdominal CT scans of the patients have not been reported by the authors, it would be interesting to know whether there has been any evidence to suggest the presence of mesenteric injuries or SB wall haematoma both of which could affect the blood circulation of a part of the bowel in the other four cases. 
In case of late post-traumatic SB perforation or stricture, it is possible that the minor initial signs go subtle for some time (especially in the presence of distracting injuries); BAT would be trivial enough to be forgotten or due to the length of posttrauma time, the patient/physician would not assume the causative relationship between the previous trauma and the current symptoms.

We are currently lacking longitudinal follow-up studies on patients with mesenteric haematoma/tear who have minimal clinical findings on initial presentation to know what percentage of such patients will re-present with clinical symptoms that warrant surgical exploration. Based on our experience and other reported cases in the literature, we propose that post-traumatic SB perforation and/or stricture should be considered in the differential diagnosis of patients who experience a delayed presentation of SB perforation/stricture following BAT. While haemodynamically stable, asymptomatic, post-trauma patients with a mesenteric tear and/or haematoma should generally be followed up and managed conservatively by the trauma team after BAT. Both the surgical team and the patient should be aware of the possible delayed complications of such injuries. We would like to thank the authors for this interesting case series highlighting the importance and the challenging situation of diagnosing the post-traumatic delayed SB stricture and perforations.

\section{References}

1. Lalwani S, Gupta M, Varma V, Mehta N, Kumaran V, Nundy S. Small bowel stricture following blunt abdominal trauma. Trop Gastroenterol. 2011;32:304-8.

2. Northcutt A, Hamidian Jahromi A, Johnson L, Youssef AM. Unusual late occurrence of bowel obstruction following blunt abdominal trauma. J La State Med Soc. 2011;163:305-7.

3. Hamidian Jahromi A, Johnson LW, Youssef AM. Delayed small bowel perforation following blunt abdominal trauma: case report and review of the literature. Asian J Surg. 2013 (In press). 